\title{
MICROALGAS COMO ADITIVOS DE DESCONTAMINAÇÃO DE AMBIENTES AQUÁTICOS: UMA REVISÃO ${ }^{1}$
}

\author{
MICROALGAS AS ADDITIVES FOR THE DECONTAMINATION OF \\ AQUATIC ENVIRONMENTS: A REVIEW
}

\author{
Aline Rossato ${ }^{2}$, Pâmella Schramm Oliveira ${ }^{3}$ e Michele Rorato Sagrillo ${ }^{4}$
}

\section{RESUMO}

A contaminação por poluentes aquáticos é considerada um dos maiores problemas ambientais, principalmente em áreas onde há influência das atividades humanas. Além disso, os efeitos desta contaminação induzem perigos a saúde pública, pois estes podem permanecer durante um longo tempo na natureza, resultando em exposição ambiental via cadeia alimentar bastante alta para consumidores de níveis tróficos elevados, incluindo os seres humanos. Deste modo, há uma necessidade de desenvolvimento de sistemas eficientes de desintoxicação de poluentes. Paralelamente, o emprego de microalgas vem sendo estudado como forma de tratamento de águas residuais de inúmeros processos industriais, para a detoxificação biológica e remoção de metais pesados e como bioindicadores na detecção de substâncias tóxicas. Neste contexto, este estudo teve como objetivo realizar uma revisão de literatura referente a aplicação de microalgas no tratamento e descontaminação de ambientes aquáticos e discutir os principais resultados encontrados. A pesquisa foi realizada na base de dados PubMed, onde os descritores utilizados foram "microalgae bioremediation contaminants". A partir dos artigos pesquisados, foi constatado que microalgas possuem a capacidade de remover diferentes poluentes aquáticos, demonstrando resultados promissores para a utilização destas no tratamento e descontaminação de ambientes aquáticos contendo compostos citotóxicos, principalmente a espécie Chlorella vulgaris.

Palavras-chave: Poluentes aquáticos, biorremediação, algas, Chlorella vulgaris.

\section{ABSTRACT}

Contamination by aquatic pollutants is considered one of the greatest environmental problems, especially in areas where human activities are influenced. Also, the effects of this contamination induce public health hazards, as these can remain for a long time in nature, resulting in environmental exposure via the food chain quite high for consumers of high trophic levels, including humans. Thus, there is a need to develop efficient systems for detoxifying pollutants. In parallel, the use of microalgae has been studied as a form of wastewater treatment of numerous industrial processes, for biological detoxification and removal of heavy metals, and as bioindicators in the detection of toxic substances. In this context, this study aimed to conduct a literature review regarding the application of microalgae in the treatment and decontamination of aquatic environments and discuss the main results found. The research was conducted in the PubMed database, where the descriptors used were "microalgae bioremediation contaminants". From the researched articles, it was found that microalgae can remove different aquatic pollutants, demonstrating promising results for their use 1 Revisão de Literatura realizada no Programa de Pós-Graduação em Nanociências.

2 Doutoranda no Programa de Pós-graduação em Nanociências - Universidade Franciscana. E-mail: alinerossato07@ gmail.com

3 Engenheira Biomédica e Mestre em Nanociências e Nanotecnologia pela Universidade Franciscana. E-mail: schramm.pso@ gmail.com

4 Contribuidora. Professora adjunta do Programa de Pós-Graduação em Nanociências - Universidade Franciscana. E-mail: sagrillorm18@gmail.com 
in the treatment and decontamination of aquatic environments containing cytotoxic compounds, especially the species Chlorella vulgaris.

Keywords: Water pollutants, bioremediation, algae, Chlorella vulgaris.

\section{INTRODUÇÃO}

A contaminação por poluentes aquáticos é considerada um dos maiores problemas ambientais, principalmente em áreas onde há influência das atividades humanas. Os resíduos de compostos farmacêuticos, por exemplo, são onipresentes em ambientes aquáticos e os métodos analíticos atuais identificam que o espectro destes está cada vez maior (HUERTA et al., 2016; XIONG et al., 2016). Os poluentes aquáticos como metais pesados e pesticidas causam elevada mortalidade de organismos aquáticos, o que pode diminuir significativamente sua população, reduzindo então a oferta de umas das fontes de proteína animal de elevada importância na nutrição humana (MALDONADO; WENDLING, 2009).

Estudos recentes realizados por Maldonado e Wendling (2019) constataram que a adição de produtos naturais na dieta para peixes pode ser uma interessante alternativa para reduzir a mortalidade e os efeitos tóxicos dos contaminantes aquáticos. Deste modo, terapias alternativas baseadas em produtos naturais estão sendo consideradas como uma nova abordagem a fim de prevenir ou reduzir os efeitos negativos dos contaminantes sobre a biota aquática, e consequentemente na saúde humana.

Dentre estas terapias, as microalgas têm demonstrado potencial para desintoxicar poluentes de águas residuais (SURTHERLAND; RALPH, 2019), devido apresentarem capacidade de adsorção, alta relação superfície/volume, ampla faixa de distribuição, metabolismo rápido, baixo custo e disponibilidade em abundância natural nos mares e oceanos (ESCAPA et al., 2017a; ESCAPA et al., 2017b; TIKOO; SCRAGG; SHALES, 1999; WANG et al., 2019). Além disso, estas são vantajosas por fornecerem um efluente de alta qualidade, bem como, devido à recuperação de recursos da biomassa de algas, para uso como fertilizante, como fonte de biocombustíveis ou de produtos como plástico, antioxidante, pigmento, glicerol etc. (CRAGGS; SUTHERLAND; CAMPBELL, 2012; MATAMOROS et al., 2016).

Portanto, este estudo teve como objetivo realizar uma revisão de literatura de artigos de pesquisas relacionados a aplicação de microalgas no tratamento e descontaminação de ambientes aquáticos, bem como, comparar os resultados encontrados para identificar quais as espécies de microalgas mais utilizadas. 


\section{MATERIAIS E MÉTODOS}

Este estudo é uma revisão da literatura, realizada entre agosto e novembro de 2020, baseada na base de dados PubMed. A busca no banco de dados foi delimitada para artigos de pesquisas utilizando os descritores "microalgae bioremediation contaminants", sem limitações de ano. A relação de documentos encontrados por ano, com estes descritores, está apresentada no gráfico da Figura 1.

Figura 1 - Documentos encontrados na plataforma PubMed utilizando os descritores "microalgae bioremediation contaminats" e seus respectivos anos.

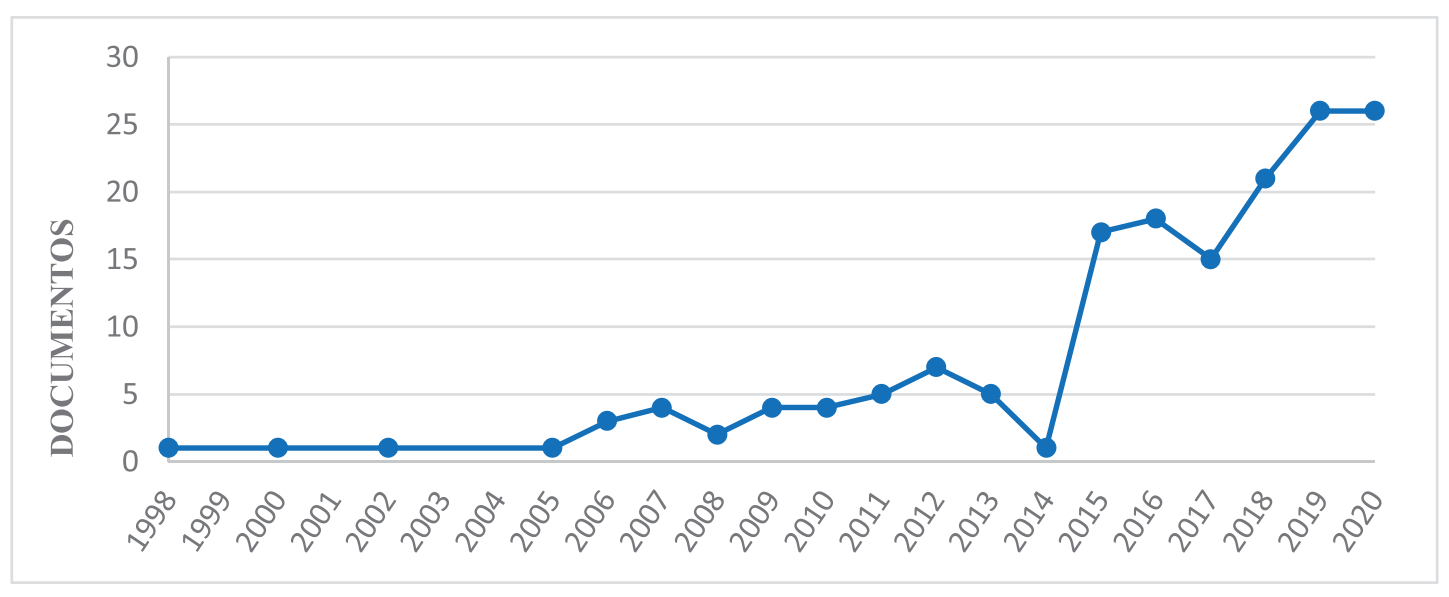

Fonte: adaptado de PubMed.

\section{RESULTADOS E DISCUSSÕES}

Na revisão de literatura realizada na plataforma PubMed, foram encontrados 143 artigos com os descritores "microalgae bioremediation contaminats". Após a análise destes documentos, muitos foram excluídos por se tratar de capítulo de livro ou artigo de revisão ou por não abordarem especificamente a biorremediação de poluentes aquáticos por microalgas. Desses, 19 artigos foram utilizados na revisão, conforme resumo apresentado na Tabela 1, pois eram os artigos de pesquisa cujo tema estava relacionado a este estudo.

Tabela 1 - Artigos de pesquisa utilizados no estudo.

\begin{tabular}{lr}
\hline \multicolumn{1}{c}{ TÍTULO } & REFERENCIA \\
\hline $\begin{array}{l}\text { Biosorption of lead(II) from aqueous solutions by non-living algal biomass Oedogonium sp. and } \\
\text { Nostoc sp. - A comparative study }\end{array}$ & GUPTA; RASTOGI, 2008 \\
\hline $\begin{array}{l}\text { Nitrogen and phosphorus removal from municipal wastewater effluent using microalgal biofilms } \\
\text { Removal and biodegradation of nonylphenol by immobilized Chlorella vulgaris }\end{array}$ & BOELEE et al., 2011 \\
$\begin{array}{l}\text { Simultaneous removal of inorganic and organic compounds in wastewater by freshwater green } \\
\text { microalgae }\end{array}$ & ZHOU et al., 2014 \\
Bioremoval of the azo dye Congo Red by the microalga Chlorella vulgaris & HERNÁNDEZ-ZAMORA \\
Removal and Biodegradation of Nonylphenol by Four Freshwater Microalgae & et al., 2015 \\
\hline
\end{tabular}




\begin{tabular}{|c|c|}
\hline Effect of microalgal treatments on pesticides in water & HULTBERG et al., 2016 \\
\hline $\begin{array}{l}\text { Biodegradation of carbamazepine using freshwater microalgae Chlamydomonas mexicana and } \\
\text { Scenedesmus obliquus and the determination of its metabolic fate }\end{array}$ & XIONG et al., 2016 \\
\hline $\begin{array}{l}\text { An oleaginous filamentous microalgae Tribonema minus exhibits high removing potential of } \\
\text { industrial phenol contaminants }\end{array}$ & CHENG et al., 2017 \\
\hline $\begin{array}{l}\text { Removal of pharmaceutical pollutants from synthetic wastewater using chemically modified } \\
\text { biomass of green alga Scenedesmus obliquus }\end{array}$ & ALI et al., 2018 \\
\hline $\begin{array}{l}\text { The effect of heavy metals on the viability of Tetraselmis marina AC16-MESO and an evaluation of } \\
\text { the potential use of this microalga in bioremediation }\end{array}$ & CAMERON et al., 2018 \\
\hline $\begin{array}{l}\text { Zebrafish embryo bioassays for a comprehensive evaluation of microalgae efficiency in the removal } \\
\text { of diclofenac from water }\end{array}$ & ESCAPA et al., 2018 \\
\hline $\begin{array}{l}\text { Optimized treatment of wastewater containing cytotoxic drugs by living and dead biomass of the } \\
\text { freshwater microalga, Chlorella vulgaris }\end{array}$ & HABIBZADEH et al., 2018 \\
\hline $\begin{array}{l}\text { Optimal strategies for bioremediation of nitrate-contaminated groundwater and microalgae } \\
\text { biomass production. }\end{array}$ & REZVANI et al., 2018 \\
\hline $\begin{array}{l}\text { Biosorption and Biodegradation of the Environmental Hormone Nonylphenol By Four Marine } \\
\text { Microalgae }\end{array}$ & WANG et al., 2019 \\
\hline Toxicity of sulfamethazine and sulfamethoxazole and their removal by a green microalga & XIONG et al., 2019 \\
\hline $\begin{array}{l}\text { Sulfonamides-induced oxidative stress in freshwater microalga Chlorella vulgaris: Evaluation of } \\
\text { growth, photosynthesis, antioxidants, ultrastructure, and nucleic acids }\end{array}$ & CHEN et al., 2020 \\
\hline $\begin{array}{l}\text { Green Microalgae Scenedesmus obliquus: Utilization for the Adsorptive Removal of Nonsteroidal } \\
\text { Anti-Inflammatory Drugs (NSAIDs) from Water Samples }\end{array}$ & SILVA et al., 2020 \\
\hline $\begin{array}{l}\text { Bioremediation and biomass production of microalgae cultivation in river water contaminated } \\
\text { with pharmaceutical effluent. }\end{array}$ & SINGH et al., 2020 \\
\hline
\end{tabular}

Fonte: construção das autoras.

Gupta e Rastogi (2008) realizaram um estudo que relata a capacidade de microalgas de água doce não vivas (secas), Oedogonium sp. e Nostoc sp., na remoção de chumbo (Pb) (II) de soluções aquosas em sistema de lote sob faixa variável de $\mathrm{pH}$ (2,99-7,04), tempo de contato (5-300 min), dose de biossorvente (0,1-0,8 g / L) e concentrações iniciais de íons metálicos (100 e $200 \mathrm{mg} / \mathrm{L})$. As condições ideais para a biossorção de $\mathrm{Pb}$ são quase as mesmas para ambas as biomassas de algas, no entanto, Oedogonium sp. foi considerado mais adequado para o desenvolvimento de um biossorvente eficiente, pois apresentou maiores valores quantidades de chumbo adsorvido na biomassa de algas no equilíbrio e tempo, e maior capacidade de adsorção. Os dados de equilíbrio se ajustaram melhor nas isotermas de Langmuir do que na isoterma de Freundlich, provando assim, a adsorção em monocamada de $\mathrm{Pb}$ em ambas as biomassas de algas. Ademais, concluiu-se que ambos os biossorventes podem ser regenerados usando 0,1 solução de $\mathrm{HCl}$ mol/ L, com recuperação de até 90\%. Os biossorventes foram reutilizados em cinco ciclos de biossorção-dessorção sem uma perda significativa na capacidade de biossorção. Sendo assim, foi constatado que ambas as biomassas algais têm potencial para serem utilizada como um biossorvente ecológico e econômico para o tratamento de efluentes portadores de $\mathrm{Pb}$ (II).

Boelee et al. (2011), analisaram a capacidade de biofilmes microalgais como uma etapa de pós-tratamento para o efluente de estações de tratamento de águas residuais municipais. Estes biofilmes foram cultivados em células de fluxo com diferentes cargas de nutrientes sob iluminação contínua 
de $230 \mu \mathrm{mol} / \mathrm{m}^{2} / \mathrm{s}$ (fótons PAR, 400-700 nm). Verificou-se que a capacidade máxima de captação do biofilme microalgal foi alcançada com taxas de carregamento de $1,0 \mathrm{~g} / \mathrm{m}^{2} /$ dia de nitrogênio e $0,13 \mathrm{~g} / \mathrm{m}^{2} /$ dia de fósforo. Estas capacidades de absorção foram as cargas mais altas nas quais os valores de efluente alvo de 2,2 $\mathrm{mg} / \mathrm{L}$ de nitrogênio $(\mathrm{N})$ e $0,15 \mathrm{mg} / \mathrm{L}$ de fósforo (P) foram alcançados. A análise da biomassa microalgal revelou um conteúdo crescente de $\mathrm{N}$ e $\mathrm{P}$ com taxas de carregamento crescentes até as capacidades máximas de absorção. A proporção interna de N:P diminuiu de 23:1 para 11:1 ao aumentar a taxa de carregamento. Esta combinação de resultados demonstrou que biofilmes microalgais podem ser usados para remover $\mathrm{N}$ e $\mathrm{P}$ de efluentes de águas residuais municipais.

Zhou et al. (2014), investigaram a remoção simultânea de vários contaminantes orgânicos e inorgânicos, incluindo nitrogênio total (TN), fósforo total (TP), metais, produtos farmacêuticos e de cuidados pessoais, produtos químicos EDCs e atividade estrogênica em águas residuais por quatro espécies de microalgas verdes de água doce, Chlamydomonas reinhardtii (C. reinhardtii), Scenedesmus obliquus (S. obliquus), Chlorella pyrenoidosa e Chlorella vulgaris (C. vulgaris). Após o tratamento por 7 dias, $76,7-92,3 \%$ de TN e 67, 5-82,2\% de TP foram removidos pelas microalgas. Segundo os autores, estas espécies de microalgas foram capazes de remover a maioria dos metais de forma eficiente (> 40\%), no entanto mostram baixa eficiência na remoção de chumbo, níquel e cobalto. Além disso, as microalgas testadas foram eficientes na remoção da maioria (> 50\%) dos compostos orgânicos selecionados e na atividade estrogênica com eficiências de remoção variando de 46,2 a 81,1\% do efluente. Portanto, foi verificado que as estas microalgas podem ser aproveitadas para remover simultaneamente vários contaminantes nas águas residuais.

Cameron e colaboradores (2018) avaliaram o comportamento e a viabilidade de Tetraselmis marina AC16-MESO no tratamento de água isolada na costa de Antofagasta, Chile. A partir deste estudo, foi descoberto que esta espécie é tolerante e capaz de remover altas concentrações de íons metálicos, em alta velocidade, em um tempo relativamente curto e com alta eficiência de sedimentação. Essas características fazem desta microalga uma candidata promissora de modelo para uso de microalgas na biorremediação de águas contaminadas com cobre, ferro e manganês.

Hernández-Zamora et al. (2015) investigaram a capacidade de remoção do corante Vermelho Congo (CR), resíduo da indústria têxtil, via tratamento com biomassa de C. vulgaris. Por meio de processos de biossorção e biodegradação, a microalga foi capaz de remover 83 e $58 \%$ do corante nas concentrações de 5 e $25 \mathrm{mg} / \mathrm{L}$, respectivamente. Além disso, o teste de toxicidade aguda (48 H) com duas espécies de cladóceros indicou que a toxicidade do corante no efluente diminuiu significativamente em comparação com as concentrações iniciais no afluente, sendo Daphnia magna a espécie menos sensível ao corante. Sendo assim, C. vulgaris reduziu significativamente a concentração e a toxicidade do corante, ou seja, pode ser uma opção viável para o tratamento desse tipo de efluente.

O efeito da microalga $C$. vulgaris em uma ampla gama de diferentes pesticidas na água foi estudado por Hultberg et al. (2016). Os tratamentos incluíram exposição de curto prazo (1 H) 
à biomassa de microalgas vivas e mortas e exposição de longo prazo (4 dias) a microalgas em crescimento ativo. A concentração inicial do pesticida foi de $63,5 \pm 3,9 \mu \mathrm{g} / \mathrm{L}$. Não houve redução significativa da quantidade total de pesticidas após a exposição de curto prazo, já para a exposição de longo prazo nas microalgas em crescimento foi alcançado uma redução significativa (concentração final 29,7 $\pm 1,0 \mu \mathrm{g} / \mathrm{L})$ em comparação com o controle $(37,0 \pm 1,2 \mu \mathrm{g} / \mathrm{L})$. Dos 38 pesticidas testados, as concentrações de 10 foram significativamente reduzidas no tratamento de microalgas de longo prazo. Um alto impacto de fatores abióticos como luz solar e aeração para redução de pesticidas foi observado quando o controle inicial e o controle de longo prazo foram comparados. Estes resultados sugerem que o tratamento de água com microalgas, habitantes naturais de águas superficiais poluídas, poderia ser mais explorado não apenas para a remoção de nutrientes inorgânicos, mas também para a remoção de poluentes orgânicos na água.

Rezvani et al. (2018) investigaram os parâmetros operacionais otimizados para remoção de nutrientes e produção de biomassa por C. reinhardtii, C. vulgaris e Ettlia sp. usando monocultivo e cultivo misto em águas subterrâneas contaminadas com nitrato. O maior volume celular e capacidade de absorção de nutrientes de Ettlia sp. foram identificados como os principais motivos para seu crescimento mais rápido, maior remoção de nutrientes e maior produção de biomassa e eficiência de sedimentação durante o tempo mínimo de retenção de 4 dias. As condições de claro-escuro também foram consideradas adequadas para a remoção de nitrato-nitrogênio, fosfato-fósforo $\left(\mathrm{PO}_{4}{ }^{3-}\right)$ e demanda química de oxigênio. Os resultados provaram que Ettlia sp. o consórcio dominante biorremedia a água subterrânea em 3 dias em comparação com outras culturas mistas e monoculturas na presença de $10 \mathrm{mg} / \mathrm{L}_{\text {de }} \mathrm{PO}_{4}^{3-}$. Além disso, a modulação do $\mathrm{pH}$ demonstrou aumentar a eficiência de sedimentação das microalgas misturadas em uma biorremediação em larga escala de águas subterrâneas.

Foi relatado pela primeira vez no estudo de Cheng et al. (2017) que uma microalga filamentosa oleaginosa Tribonema minus (T. minus) exibiu forte capacidade de remoção de fenol ambiental. Segundo os autores, os filamentos desta microalga mostraram 449,46 mg / g de capacidade de captação de fenol, obviamente maior do que aquelas cepas com baixa absorção de fenol, como Scenedesmus dimorphus. Além disso, os fenóis puderam ser removidos com eficiência na concentração inicial de fenol de até $700 \mathrm{mg} / \mathrm{L}$. Simultaneamente, por meio do crescimento de T. minus, a concentração de fenol pôde ser diminuída de $100 \mathrm{mg} / \mathrm{L}$ para a faixa de $0,1-0,5 \mathrm{mg} / \mathrm{L}$, que atende às necessidades de descarte industrial de contaminantes de fenol na maioria dos países. Assim, T. minus é uma espécie de algas em potencial para ajudar na construção de um processo integrado para a produção de biomassa oleaginosa e biorremediação de contaminantes fenólicos.

O composto nonilfenol (NP) é encontrado em muitos produtos industriais, domésticos, agrícolas, cosméticos e farmacêuticos, ou seja, está presente em grande quantidade no nosso dia a dia. Neste contexto, Gao e colaboradores (2011) compararam a capacidade de remoção e biodegradação do NP por células imobilizadas com alginato de $C$. vulgaris e suas respectivas culturas livres. Embora 
a imobilização tenha diminuído significamente a taxa de crescimento e a eficiência de biodegradação de NP, foi constatado que após o aprimoramento por meio de otimização de concentração de biomassa, estes foram mais eficazes na remoção de NP do que as células livres. Portanto, estes resultados demonstraram que células imobilizadas de C. vulgaris sob biomassa ideal e condições fotoautotróficas são eficazes na remoção de NP de água contaminada.

He e colaboradores (2016) estudaram a remoção e biodegradação de NP por quatro microalgas de água doce, em culturas livres de bactérias expostas a diferentes concentrações de NP durante 5 dias. As taxas de remoção de NP foi de Ankistrodesmus acicularis (A. acicularis ) (83,77\%), seguida por Chlorella vulgaris (80,80\%), Scendesmus quadriauda (S. quadriauda) (70,96\%) e Chroococcus minutus (C. minutus) (64,26\%), enquanto as taxas de biodegradação foram C. vulgaris $(68,80 \%)>$ A. acicularis $(65,63 \%)>S$. quadriauda $(63,10 \%)>C$. minutus $(34,91 \%)$. Além disso, o conteúdo extracelular de NP foi menor do que o conteúdo intracelular em todas as algas testadas, ou seja, a remoção de NP do meio foi principalmente devido à degradação das algas. Esses resultados indicaram que $A$. acicularis e $C$. vulgaris são mais tolerante a NP e poderiam ser usadas para tratamento de sistemas aquosos contaminados por NP de forma eficaz por bioremoção e biodegradação.

Em outro estudo relacionado a NP, Wang e colaboradores (2019) investigaram a absorção intracelular, adsorção extracelular e biodegradação deste composto por quatro espécies de microalgas marinhas (Phaeocystis globosa, Nannochloropsis oculata, Dunaliella salina e Platymonas subcordiformis). Deste modo, constataram uma redução acentuada de NP em meio contendo as microalgas testadas durante as primeiras $24 \mathrm{H}$ de incubação, bem como, essas espécies exibiram a maior capacidade de adsorção e absorção em 24h de cultivo. Entretanto, a quantidade de NP absorvida e adsorvida pelas microalgas diminuiu com o aumento do tempo de cultura e a absorção intracelular foi maior do que a adsorção extracelular. Além disso, foi observado que após $120 \mathrm{H}$ de exposição, as espécies biodegradaram a maior parte de NP do meio, com eficiências variando de 43,43 a 90,94\%. Portanto, neste estudo foi constatado que as microalgas testadas possuem altas porcentagens de biodegradação e podem, assim, melhorar a biorremediação de água contaminada com NP.

Xiong et al. (2016) avaliou a toxicidade, o estresse celular e biodegradação da carbamazepina (CBZ) em Chlamydomonas mexicana (C. mexicana) e S. obliquus. O crescimento de ambas as espécies de microalgas diminuiu com o aumento da concentração de CBZ. O crescimento de S. obliquus foi inibido significativamente $(97 \%)$ a $200 \mathrm{mg} / \mathrm{L}$ de CBZ, em comparação com o controle após 10 dias; enquanto C. mexicana apresentou $30 \%$ de inibição nas mesmas condições experimentais. Características bioquímicas, incluindo clorofila total, conteúdo de carotenóides e atividades enzimáticas para ambas as espécies foram afetadas por CBZ em concentração relativamente alta. C. mexicana e S. obliquus atingiram um máximo de $35 \%$ e $28 \%$ de biodegradação de CBZ, respectivamente. Dois metabólitos (10,11-diidro-10, 11-expoxicarbamazepina e n-hidroxi-CBZ) foram identificados por cromatografia líquida de ultra-performance Orbitrap e espectrometria de massa, como 
resultado da biodegradação de $\mathrm{CBZ}$ por $C$. mexicana. Por fim, este estudo demonstrou que $C$. mexicana foi mais tolerante à $\mathrm{CBZ}$ e pode ser utilizada para tratamento de águas residuais contaminadas com CBZ.

Xiong e colaboradores (2019) avaliaram a capacidade de S. obliquus na biodegradação e remoção de uma mistura de sulfametazina (SMZ) e sulfametoxazol (SMX). A microalga demonstrou-se capaz de biodegradar os produtos químicos testados, bem como, eficiente ao remover da mistura 31,4-62,3\% de SMZ (0,025-0,25 mg / L) e 27,7-46,8\% de SMX (0,025-0,25 mg / L), após 12 dias de cultivo. Além disso, foi observado que a biodegradação foi maior em concentrações mais altas de SMZ e SMX, indicando assim, que a biodegradação microalgal pode ser um mecanismo eficiente de adaptação de microalgas aos antibióticos.

Recentemente, Chen e colaboradores (2020) relataram as mudanças fisiológicas de C. vulgaris na presença de altas concentrações de três sulfonamidas (SAs) de estruturas semelhantes (sulfadiazina (SD), sulfamerazina (SM1) e sulfametazina (SM2)) e determinaram a sua capacidade de remover as mesmas após exposição de 7 dias. SD, SMI e SM2 inibiram o crescimento de C. vulgaris em 7,9-22,6\%, 7,2-45,9\% e 10,3-44\%, respectivamente. A ultraestrutura e o DNA das células das microalgas tratadas com SAs foram afetados, como em mudanças consideráveis na parede celular, cloroplasto e mitocôndria e na migração de DNA. Além disso, a capacidade de C. vulgaris na remoção dos antibióticos testados foi de 29\% para SD, 16\% para SM1 e 15\% para SM2. Os resultados encontrados sugerem que certas concentrações de antibióticos específicos podem induzir o crescimento de algas, assim como, o processo de biodegradação mediado por algas pode acelerar na remoção destes contaminantes.

O desempenho adsortivo da biomassa de S. obliquus foi investigado na remoção de ácido salicílico e ibuprofeno, por Silva e colaboradores (2020). Além disso, a biomassa de microalgas foi caracterizada antes e após a adsorção desses fármacos, a fim de relacionar os resultados obtidos na sua remoção com as propriedades da biomassa e para identificar se a adsorção resultou em alguma modificação. Ajustes dos resultados de adsorção cinética para a equação de pseudo-segunda ordem indicaram que as taxas de adsorção na biomassa de microalgas eram semelhantes às do carvão ativado comercial usado como referência. Os resultados de equilíbrio foram descritos pelo modelo de isoterma de Langmuir e as capacidades máximas de adsorção ajustadas para ácido salicílico e ibuprofeno foram menores quando comparados ao do carvão ativado comercial. Embora as microalgas apresentarem capacidades menores, estas podem ser um adsorvente sustentável alternativo, pois a adsorção dos medicamentos anti-inflamatórios não esteroidais foi favorável, espontânea e exotérmica, bem como, não induziu nenhuma modificação significativa nos grupos de superfície da biomassa de microalgas após a adsorção dos fármacos. Os dados obtidos neste trabalho apontam para a utilização de biomassa de microalgas mortas não modificadas como um adsorvente verde alternativo para a remoção de fármacos.

Escapa et al. (2018) avaliaram a eficiência das microalgas Chlorella sorokiniana, C. vulgaris e S. obliquus na biorremediação de água contaminada com diclofenaco. Estas foram cultivadas 
em fotobiorreatores de coluna borbulhante (PBRs) em batelada até o final da fase de crescimento exponencial. A concentração de diclofenaco no meio aquático PBRs diminuiu ao longo do crescimento de todas as microalgas, o que indica a biodegradação como o principal mecanismo de remoção. Entre as cepas testadas, S. obliquus foi o mais eficaz na remoção de diclofenaco ( $99 \%$ de uma concentração inicial de $25.000 \mu \mathrm{g} / \mathrm{L}$ ). Devido a remoção ser tão grande, os efluentes finais dos PBRs foram testados quanto aos seus efeitos no desenvolvimento embrionário do peixe-zebra. Novamente, para S. obliquus o tratamento foi o mais eficiente na redução da toxicidade, não havendo efeito dos efluentes correspondentes sobre a mortalidade embrionária ou incidência de anomalias. Nas três cepas os efeitos de toxicidade dos efluentes foram iguais ou inferiores aos determinados para soluções de diclofenaco com a mesma concentração. Sendo assim, ao final da cultura em lote, a remoção do diclofenaco pelas microalgas testadas não envolveu a geração de TPs tóxicos para embriões de peixe-zebra.

Habibzadeh et al. (2018) avaliaram a eficiência da biomassa viva e morta de C. vulgaris na biorremediação em efluentes do fármaco anticâncer Flutamida (FLU). A microalga viva apresentou melhor desempenho na remoção de FLU considerando a quantidade de biomassa, pH e tempo de adsorção, bem como, apresentou maior capacidade máxima de sorção (26,8 mg / g) que a biomassa morta (12,5 mg / g). Uma abordagem de regressão não linear revelou que as isotermas de Freundlich e Langmuir poderiam se ajustar bem aos dados de adsorção. Estudos cinéticos mostraram que o processo de adsorção por microalgas vivas e mortas seguiu o modelo modificado de Freundlich. A otimização do processo pela metodologia de superfície de resposta indicou que FLU na concentração de $50 \mu \mathrm{M}$, pH 7,4 e 10 min poderia ser removido em até 98,5\% pela biomassa viva. Estes resultados demonstram que a utilização da microalga pode ser uma tecnologia promissora para o tratamento de águas residuárias contendo compostos citotóxicos.

Ali et al. (2018) estudaram a remoção de poluentes farmacêuticos de águas residuais sintéticas usando biomassa quimicamente modificada de alga verde $S$. obliquus. A biomassa desta foi modificada em solução alcalina e utilizada para a biossorção de tramadol (TRAM) e outros fármacos. Os resultados revelaram alta capacidade de adsorção do TRAM sobre a biomassa algal modificada (MAB) após 45 min, com porcentagem de remoção de 91\%. A biossorção de TRAM na MAB prossegue com o modelo de isoterma de Freundlich com coeficiente de correlação $(0,942)$, o que mostra que a absorção foi conduzida por quimissorção. Na matriz farmacêutica, as capacidades de adsorção registradas para TRAM, Cefadroxil (CEFA), Paracetamol (PARA), Ciprofloxacina (CIP) e Ibuprofeno (IBU) são 68, 58, 42, 42 e 39 mg / g sobre MAB em pH natural e dose de MAB de 0,5 g / L. Além disso, o consumo de oxigênio pelas bactérias foi aplicado para estimar a toxicidade do fármaco, indicando a reutilização eficiente da biomassa de algas modificadas para biossorção de produtos farmacêuticos, sendo que diminuiu apenas 4,5\% da eficiência de adsorção após três execuções. Desse modo, constatou-se que MAB é um adsorvente reutilizável promissor para descontaminação de águas residuais de produtos farmacêuticos. 
Recentemente, Singh e colaboradores (2020) avaliaram o potencial de microalgas de Chlorella sp. SL7A, Chlorococcum sp. SL7B e Neochloris sp. SK57 cultivadas em água de rio contaminada com efluentes farmacêuticos para produção de biomassa e lipídios. Neste estudo, os autores observaram que a espécie de rápido crescimento neste meio foi Neochloris sp. SK57. Já a biomassa máxima e o rendimento lipídico foram obtidos por Neochloris sp. SK57 (0,52 g / L) e Chlorococcum sp. SL7B $(0,129 \mathrm{~g} / \mathrm{L})$. Este aumento da biomassa e lipídios pode ser devido à assimilação de nutrientes orgânicos e estresse causados a outros componentes presentes na água do rio. O perfil de ácidos graxos saturados da biomassa de algas foi aumentado em óleos de Neochloris sp. SK57, bem como, a sua adequação em aplicações de alimentos e combustíveis. Por fim, foi observada uma melhora significativa da qualidade da água do rio após o cultivo das microalgas.

\section{CONCLUSÃO}

A poluição do ecossistema aquático por poluentes orgânicos e inorgânicos, industriais, pesticidas ou fármacos têm sido considerada um grave problema, pois a presença de contaminantes citotóxicos recalcitrantes em ambientes aquáticos apresentam efeitos prejudiciais significativos aos ecossistemas e à saúde humana. Baseado nisto, foi realizado um estudo de literatura sobre abordagens alternativas de tratamentos de efluentes utilizando microalgas. Nos resultados encontrados, C. mexicana, S. obliquus, C. vulgaris e Neochloris sp. demonstraram serem as microalgas mais indicadas para remoção de fármacos, enquanto $C$. vulgaris foi a mais estudada para descontaminação de poluentes orgânicos e inorgânicos. Em poluentes pesticidas, apenas C. vulgaris foi utilizada, ou seja, constatou-se a necessidade de mais pesquisas nesta área para melhor compreensão da eficácia do tratamento de efluentes frente a estes contaminantes utilizando microalgas. Portanto, nesta revisão foi verificado que o uso de microalgas (especialmente $C$. vulgaris) como descontaminantes aquáticos é promissor, no entanto, a eficiência de remoção destes tratamentos está relacionada a espécie de microalga utilizada e o tipo de poluente tratado.

\section{REFERENCIAS}

ALI, M. E. M. et al. Removal of pharmaceutical pollutants from synthetic wastewater using chemically modified biomass of green alga Scenedesmus obliquus. Ecotoxicology and Environmental Safety, v. 151, p. 144 - 152, 2018.

BOELEE, N. C. et al. Nitrogen and phosphorus removal from municipal wastewater effluent using microalgal biofilms. Water Research, v. 45, n. 18, p. 5925-5933, 2011. 
CAMERON, H. et al. The effect of heavy metals on the viability of Tetraselmis marina AC16-MESO and an evaluation of the potential use of this microalga in bioremediation. PeerJ Journals, v.6, p. 1-15, 2018.

CHEN, S. et al. Sulfonamides-induced oxidative stress in freshwater microalga Chlorella vulgaris: Evaluation of growth, photosynthesis, antioxidants, ultrastructure, and nucleic acids. Sci Rep, v. 10, e8243, 2020.

CHENG, T. et al. An oleaginous filamentous microalgae Tribonema minus exhibits high removing potential of industrial phenol contaminants. Bioresource Technology, v. 238, p. 749-754, 2017.

CRAGGS, R.; SUTHERLAND, D.; CAMPBELL, H. Hectare-scale demonstration of high rate algal ponds for enhanced wastewater treatment and biofuel production. J Appl Phycol, v. 24, p. 329-337, 2012.

ESCAPA, C. et al. Comparison of the culture and harvesting of Chlorella vulgaris and Tetradesmus obliquus for the removal of pharmaceuticals from water. Journal of Applied Phycology, v. 29, p. 1179-1193, 2017a.

ESCAPA, C. et al. Valorization of Microalgae Biomass by Its Use for the Removal of Paracetamol from Contaminated Water. Water, v. 9, e312, 2017 b.

ESCAPA, C. et al. Zebrafish embryo bioassays for a comprehensive evaluation of microalgae efficiency in the removal of diclofenac from water. Science of the total Environment., v. 640-641, n. 1, p. 1024-1033, 2018.

GAO, Q. T.; WONG, Y. S.; TAM, N. F. Y. Removal and biodegradation of nonylphenol by immobilized Chlorella vulgaris. Bioresource Technology, v. 102, n. 22, p. 10230-10238, 2011.

GUPTA, K. V.; RASTOGI, A. Biosorption of lead(II) from aqueous solutions by non-living algal biomass Oedogonium sp. and Nostoc sp.- A comparative study. Colloids and Surfaces B: Biointerfaces, v. 64 , n. 2, p. 170-178, 2008.

HABIBZADEH, M. et al. Optimized treatment of wastewater containing cytotoxic drugs by living and dead biomass of the freshwater microalga, Chlorella vulgaris. Ecological Engineering, v. 111, p. 85-93, 2018. 
HE, N. et al. Removal and Biodegradation of Nonylphenol by Four Freshwater Microalgae. Int. J. Environ. Res. Public Health, v. 13, n. 12, e1239, 2016.

HERNÁNDEZ-ZAMORA, M. et al. Bioremoval of the azo dye Congo Red by the microalga Chlorella vulgaris. Environ Sci Pollut Res, v. 22, p. 10811-10823, 2015

HUERTA, B. et al. Determination of a broad spectrum of pharmaceuticals and endocrine disruptors in biofilm from a waste water treatment plant-impacted river. Science of the Total Environment, v. 540, p. 241-249, 2016.

HULTBERG, M. et al. Effect of microalgal treatments on pesticides in water. Environmental Technology, v. 37, n.7, p. 893-898, 2016.

MATAMOROS, V. et al. Assessment of the mechanisms involved in the removal of emerging contaminants by microalgae from wastewater: a laboratory scale study. Journal of Hazardous Materials, v. 301, p. 197-205, 2016.

REZVANI, F. et al. Optimal strategies for bioremediation of nitrate-contaminated groundwater and microalgae biomass production. Environmental Science and Pollution Research, v. 25, p. 27471 $27482,2018$.

SILVA, A. et al. Green Microalgae Scenedesmus obliquus: Utilization for the Adsorptive Removal of Nonsteroidal Anti-Inflammatory Drugs (NSAIDs) from Water Samples. International Journal of Environmental Research and Public Health - Open Access Journal, v. 17, n. 10, e3707, 2020.

SINGH, A. et al. Bioremediation and biomass production of microalgae cultivation in river water contaminated with pharmaceutical effluent. Bioresource Technology, v. 307, p. 123 - 233, 2020.

SUTHERLAND, D. L.; RALPH, P. J. Microalgal bioremediation of emerging contaminants Opportunities and challenges. WATER RES. 164, e114921, 2019.

TIKOO, V.; SCRAGG, A. H.; SHALES, S. W. Degradation of Pentachlorophenol by Microalgae. Journal of Chemical Technology \& Biotechnology Biotechnology, v. 68, p. 425-431, 1999.

WANG, L. et al. Biosorption and Biodegradation of the Environmental Hormone Nonylphenol By Four Marine Microalgae. Scientific reports, v. 27, n. 1, e5277, 2019. 
XIONG, J. et al. Biodegradation of carbamazepine using freshwater microalgae Chlamydomonas mexicana and Scenedesmus obliquus and the determination of its metabolic fate. Bioresource Technology, v. 205, p. 183-190, 2016.

XIONG, J. et al. Toxicity of sulfamethazine and sulfamethoxazole and their removal by a green microalga. Scenedesmus obliquus. Chemosphere, v. 218, p. 551-558, 2019.

ZHOU, G. et al. Simultaneous removal of inorganic and organic compounds in wastewater by freshwater green microalgae. Environmental Science: Processes \& Impacts, v. 16, n. 8, p. 2018-2027, 2014. 